\title{
Der schwere Weg zum leichteren Leben
}

\begin{abstract}
_ Adipositas ist keine Lifestyle-Erkrankung, bei der betroffene Menschen durch ihr eigenes Verhalten dick werden, sondern vielmehr eine chronische, häufig progrediente Erkrankung, erklärte Prof. Arya Sharma, wissenschaftlicher Direktor des Canadian Obesity Networks, Edmonton/Kanada. Komplexe neuroendokrine Mechanismen sorgen bei der Erkrankung dafür, dass ein einmal erreichtes Übergewicht schwierig zu reduzieren ist, bzw. dass eine erzielte Gewichtsabnahme langfristig kaum gehalten wird. „Der Körper hat sich auf ein Sollgewicht eingestellt, das er dann ver-
\end{abstract}

teidigt“, so Sharma. Die Folge: Bei 95\% der Patienten scheitern gewichtsreduzierende Maßnahmen. Nur einer von $20 \mathrm{~Pa}$ tienten kann ein erreichtes geringeres Gewicht auf Dauer halten.

Um bei adipösen Patienten einen anhaltenden Therapieerfolg zu erreichen sei ein langfristiges, interdisziplinäres Therapiemanagement erforderlich, so Prof. Martina de Zwaan, Hannover. Wichtig seien realistische Therapieziele. Schon Gewichtsverluste von 5-10\% verbesserten die Stoffwechsellage deutlich.

Basis der Behandlung sind Ernährungsumstellung, Steigerung der Bewe- gung und Verhaltensänderungen, die durch medikamentöse Maßnahmen unterstützt werden können. Eine Option ist Liraglutid (Saxenda ${ }^{\circledR}$ ): Das GLP1-Analogon wirkt an spezifischen $\mathrm{Re}$ zeptoren in Gehirn, Pankreas und Gastrointestinaltrakt und reguliert den Appetit durch die Steigerung des Völleund Sättigungsgefühls sowie eine Reduzierung des Hungergefühls und des Wunsches nach Nahrung.

Michael Koczorek

- Fachpressegespräch „Keine leichte Aufgabe: Stigmata auflösen - Adipositas erfolgreich therapieren", Adipositastage 2016; Frankfurt, November 2016 (Veranstalter: Novo Nordisk)

\section{Rheumatoide Arthritis}

\section{Alle Therapieoptionen ausschöpfen}

_ In der Behandlung von Patienten mit rheumatoider Arthritis (RA) wurde in den vergangenen 20 Jahren vieles erreicht. Doch manche Patienten sprechen auf die verfügbaren Medikamente nicht ausreichend an, und andere vertragen Methotrexat (MTX) nicht gut, wodurch die Adhärenz beeinträchtigt wird. Es gibt also Bedarf an weiteren Therapieoptionen. Diese therapeutische Lücke könnte der orale Januskinase(JAK)-Inhibitor Tofacitinib schließen.

In der Pathogenese der RA spielen Zytokine eine wichtige Rolle. Sie nutzen den JAK-Signalweg, um den Entzündungsreiz ins Zellinnere weiterzugeben. Um diesen Signalweg zu unterbrechen, hat man die JAK-Inhibitoren entwickelt: Sie fangen die Zytokinsignale ab und stoppen so den Entzündungsreiz. Anders als Biologika, die aufgrund ihrer Molekülgröße gespritzt oder als Infu- sion gegeben werden, können JAKInhibitoren oral verabreicht werden.

Der JAK-Hemmer Tofacitinib wurde in den USA bereits 2012 als Monotherapie oder in Kombination mit einem konventionellen Disease-Modifying Antirheumatic Drugs (DMARD) nach MTX-Versagen zur Behandlung bei RA zugelassen. Mit der Zulassung in Deutschland wird im Laufe des Jahres gerechnet.

Um das optimale Einsatzspektrum des JAK-Hemmers besser eingrenzen zu können, haben jetzt Forscher um Dr. Christina Charles-Schoeman, Los Angeles, USA. Daten von RA-Patienten aus neun randomisierten, doppelblinden, placebokontrollierten Studien analysiert. Die Ergebnisse: Grundsätzlich wirkte der JAK-Hemmer sowohl bei DMARD-naiven Patienten, als auch bei solchen, die auf DMARD nicht aussreichend anspra-

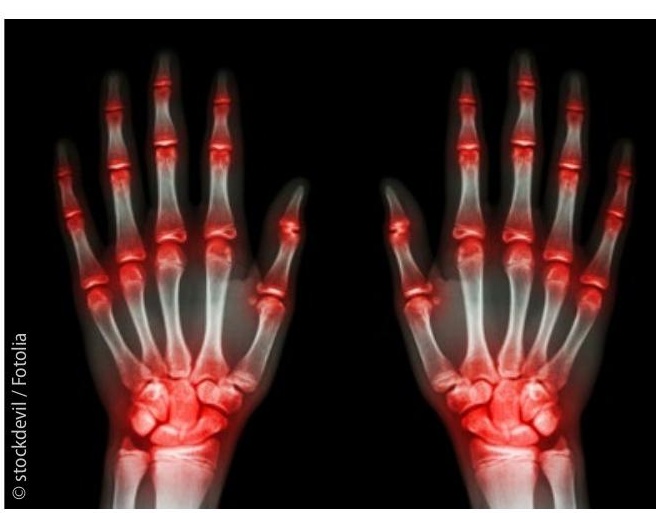

Bei rheumatoider Arthritis sind vor allem die kleinen Gelenke betroffen.

chen. Das klinische Ansprechen war aber bei den therapienaiven Patienten größer. So erreichten mit $2 \times 5 \mathrm{mg} / \mathrm{d}$ Tofacitinib $60,3 \%$ der DMARD-naiven Patienten ein ACR-20-Ansprechen (mindestens 20\%ige Verbesserung der Symptomatik), im Vergleich zu 43,4\% bei denjenigen, die auf DMARD unzureichend angesprochen hatten.

Christina Ott

- Ann Rheum Dis. 2016;75:1293-301 